\title{
INFLUÊNCIA DO TIPO DE ALIMENTO E DA TEMPERATURA NA EVACUAÇÃO GÁSTRICADA PIRANHA CAJU (Pygocentrus nattereri) EM CONDIÇÕES EXPERIMENTAIS ${ }^{1}$
}

\author{
Emerson Carlos Soares e SILVA², Carlos A. R. M. ARAÚJO-LIMA³
}

RESUMO: taxa de evacuação gástrica é uma variável importante para estimar o consumo diário de alimento e portanto a pressão de predação que um piscívoro exerce sobre as presas. Esta taxa pode ser influenciada pela temperatura, tamanho do predador e pelo tipo de alimento. Neste trabalho medimos experimentalmente os efeitos que estas três variáveis exercem sobre a evacuação gástrica de Pygocentrus nattereri, um dos principais piscívoros da Amazônia. Para isso foram utilizadas temperaturas variando de $27^{\circ}$ a $31^{\circ} \mathrm{C}$ e os seguintes itens alimentares: músculo e nadadeira de Semaprochilodus insignis e gafanhoto da espécie Paulinia sp. Estes alimentos compõem a dieta silvestre da piranha. $\mathrm{O}$ tamanho da piranha não influenciou na evacuação gástrica. Músculos obtiveram uma taxa de evacuação gástrica de $18,3 \%$ h -1, enquanto que nadadeira e gafanhoto obtiveram uma taxa média de $10,7 \% \mathrm{~h}-1$. O efeito da temperatura foi mais pronunciado quando o item alimentar era músculo do que com nadadeira e insetos.

PALAVRAS-CHAVE: Evacuação gástrica, peixes, Pygocentrus nattereri, temperatura, Amazônia.

\section{INFLUENCE OF FOOD TYPE AND TEMPERATURE ON GASTRIC EVACUATION OF Pygocentrus nattereri AND EXPERIMENTAL CONDITIONS}

ABSTRACT : The gastric evacuation rate is an important variable to measure the daily ration and feeding rhythm therefore, the predation pressure of a piscivorous fish. The evacuation can be influenced by temperature, predator size and food type. In this paper it is presented experimental data of the effect of three food types in Pygocentrus nattereri, a main piscivorous amazonian fish. The temperature ranged from 27 to $31^{\circ} \mathrm{C}$ and three food items were used (fish muscle, fish fin rays and insect). These food items are ordinarily consumed by the piranha. Predator size had no effect on gastric evacuation. Fish muscles were evacuated at rate of $18.3 \% \mathrm{~h}-1$, while fish fin and insect average ratio $10.7 \% \mathrm{~h}-1$. The gastric evacuation

\footnotetext{
${ }^{1}$ Parte de Dissertação de Mestrado FMV - USP.

${ }^{2}$ ESPAM - Escola Sperior Batista do Amazcnas. R. Rio Javary n. ${ }^{\circ} 278$, Conjunto Abŕlio Nery. Adriańpolis - Manaus - Anezonas. Email: enersonainpa.gov.br

${ }^{3}$ INEA / Instituto Nacional de Pesquisas da Amezânia. Av. André Araújo n. ${ }^{\circ} 2936$, Petrópolis, GFP: $69011-970$ Mbnaus - Amazonas.
} 
increased with temperature. The effect of temperature was more pronounced on fish muscle than in fish fin or insect.

KEYWORDS: gastric evacuation, fish, Pygocentrus nattereri, temperature, Amazon.

\section{INTRODUÇÃO}

A piranha caju (Pygocentrus nattereri Kner, 1860) é um serrasalmídeo típico de ambientes lênticos. Sua distribuição geográfica ainda é mal conhecida e há necessidade de uma melhor definiçāo dos limites de sua ocorrência, porém é uma espécie comum na Amazônia Central e no pantanal Mato-grossense (Fink, 1993).

Este predador é uma das espécies mais abundantes na várzea do rio Solimões/Amazo nas (Merona \& Bittencourt, 1993; Saint-Paul \& Zuanon, 2000) e apresenta características biológicas e demográficas que sugerem táticas eficientes à vida em lagos de várzea (Bittencourt, 1994). Machado-Allison \& Garcia (1986) observaram que P. nattereri é zooplanctófaga durante os primeiros estágios de desenvolvimento nas planícies alagadas da Venezuela, e vai alterando sua dieta conforme cresce. Foxx (1972) observou em aquário que a piranha caju alimenta-se primeiramente da nadadeira caudal de sua vítima, em seguida dos olhos. Este ataque seletivo sugere que a estratégia das pira nhas é aumentar a vulnerabilidade e reduzir a mobilidade de suas presas (Pauly, 1994). O regime alimentar natural de peixes tropicais, em particular da piranha caju, curiosamente ainda é pouco conhecido. Nos estudos de Pauly (1994), foi observado que P. nattereri tem o hábito de arrancar pedaços da presa para alimentar-se, diferente de outros piscívoros como o tucunaré por exemplo. Portanto o tamanho da presa não é fator limitante para este preda- dor. P. natttereri possui também a reputação de ser um predador considerado voraz, agressivo, e capaz de consumir rapidamente grande quantidade de presas.

A voracidade ou necessidade de um predador ingerir suas presas é refleti da pela capacidade que o indivíduo apresenta de digerir e evacuar o alimento e está ligada a suas necessidades energéticas (Sainsbury, 1986). Na maioria dos peixes o processo de digestão é iniciado no momento em que o alimento entra no estômago. As enzimas digestivas, que são controladas por nervos e sinais hormonais, agem sobre o alimento de acordo com o tempo. Conhecendo-se o peso inicial da refeiçāo e o tempo decorrido após a ingestão, podemos estimar a taxa de evacuação estomacal. $\mathrm{O}$ alimento ingerido pode ser medido em termos de peso úmido e peso seco, visto que Glenn \& Ward (1968) observaram que ambas medidas são equivalentes. Entretanto o peso úmido deve ser corrigido, pois os valores medidos por este método, tendem a superestimar valores acima de $100 \%$ (Magnusson, 1969).

O tempo de evacuação gástrica pode ser afetado pelo tipo e tamanho de presa, tamanho do predador e estresse do ambiente (Bromley, 1994). A privação de alimento, ingestão de refeições subseqüentes, combinação da composição do alimento, temperatura da água e a fisiologia do predador, também influenciam no tempo de evacuação gástrica (Jobling, 1987; Salvanes et al., 1995) .

O tipo de presa pode influenciar nas taxas e seleção de modelos de evacuação gástrica 
em peixes. Jones (1974) notou que diferentes tipos de alimentos podem ser evacuados a diferentes taxas. Tyler (1970) e Elliot (1972), em ensaios laboratoriais, observaram que a quali dade e a superfície do alimento podem influenciar na seleção e nos modelos que descrevem o esvaziamento estomacal, bem como na digestão do alimento. O tipo de tecido da presa, a textura, o formato do esqueleto, bem como pre sença ou ausência de carapaças e composiçōes dos tecidos (quantidade de carboidratos, lipídios), podem também influenciar na diges tão e, portanto, no tempo de evacuação.

Bromley (1991), analisando conteúdos estomacais de bacalhau, observou que a lagosta da Noruega (Nephrops norvegicus), foi evacuada lentamente, enquanto que um peixe (Sprattus sprattus), foi digerido rapidamente. As medusas da ordem Rhizostomea foram digeridas completamente dentro de 20 minutos, enquanto lulas (Loligo vulgaris reynaudii) e alguns peixes, levaram 11 horas para serem digeridos em temperaturas semelhantes. $\mathrm{O}$ "krill", um crustáceo marinho e alguns moluscos são digeridos mais rapidamente que poliquetas (Nelson \& Ross, 1995). Segundo Nelson \& Ross (1995), esta diferença pode estar relacionada à composição da superfície do tecido, ao teor de gordura ou ao endoesqueleto desses animais. A mesma conclusão foi alcançada por outros autores, quando examinaram a evacuação gástrica de várias espécies de peixes (Brett \& Higgs, 1970; Tyler, 1970; Elliot, 1972; Swenson \& Smith, 1973; Persson, 1979; Figueiredo \& Vieira, 1998).

A temperatura é o principal fator abiótico que influencia na taxa do metabolismo em peixes, atingindo diretamente o consumo de alimento e o processo digestivo (Smith, 1989). Éstá correlacionada positivamente com a taxa de consumo diário de diferentes tamanhos de presas (Salam \& Davies, 1994) e também afeta as taxas de alimentaçāo, a atividade hidrolítica das enzimas digestivas e as taxas de absorção intestinal. Em pesquisas realizadas com peixes de regiões temperadas, o consumo de alimento durante o dia aumentou, em conseqüência das variaçōes de temperatura (Smith, 1989). Assim a temperatura deve afetar o tempo de evacuação de P. nattereri, aumentando a velocida de de passagem do alimento conforme o aumento da temperatura. Portanto a taxa de evacuação gástrica em peixes depende de fatores ambientais, como a temperatura, e nutricionais como o tipo e qualidade do alimento.

O presente estudo pretende estimar experimentalmente a taxa de evacuação gástrica de P. nattereri, considerando a influência da tem peratura da água e do tipo de alimento.

\section{MATERIAL E MÉTODOS}

Os noventa e seis exemplares de P. nattereri foram capturados vivos no lago Jacaretinga, município do Careiro da Várzea, nos meses de maio, junho e agosto de 2001, utilizando anzol e linha de mão em vários lo cais do lago próximos a bancos de macrófitas. Após a captura, as piranhas foram acondicionadas e transportadas em uma caixa de isopor de 100L equipada com aeradores portáteis. $\mathrm{O}$ tempo de transporte durou sete horas incluin do o período de espera na caixa de isopor. Os peixes foram pré-aclimatados por mais de duas semanas antes do experimento, sendo coloca dos em três tanques circulares de 500L cuja água era recirculada através de filtros biológi cos e com aeraçāo contínua. A iluminaçāo dos tanques foi reduzida em $50 \%$ da intensidade externa usando telas de nylon para minimizar o estresse dos animais e a água foi trocada parcialmente a cada quinze dias. As temperaturas 
nos tanques de pré-aclimatação permaneceram em torno de $26^{\circ} \mathrm{C}$ e os teores de oxigênio dissolvido próximos a $5 \mathrm{mg} / \mathrm{l}$. Os experimentos de evacuação gástrica foram realizados em aquári os de 60 litros com a densidade de 1 peixe/ aquário, mantendo-se as temperaturas experimentais próximas a $27^{\circ}, 29^{\circ}$ e $31^{\circ} \mathrm{C}$, monitoradas a cada 30 minutos por termosensores acoplados a um registrador digital de quatro canais. As paredes externas dos aquários foram envolvidas com plástico negro para reduzir a percepção dos peixes em relação à movimentação externa. A aclimatação nos aquários experimentais durou uma semana, sendo os exemplares alimentados com músculos de peixes, após a qual os exemplares passaram por um jejum forçado de 48 horas para total evacuaçāo dos resíduos estomacais.

Os alimentos utilizados no experimento foram músculo de peixe, nadadeira de peixe e insetos. Estes alimentos foram escolhidos por fazerem parte da dieta da espécie, sendo os mais encontrados na sua dieta como indicado por uma análise preliminar do conteúdo estomacal de indivíduos silvestres. Foram usados músculos e nadadeiras de Semaprochilodus insignis (Schomburgk, 1841) e gafanhotos inteiros (Paulinia sp.).

Os alimentos foram pesados com precisão de até $0,001 \mathrm{~g}$ antes de serem oferecidos aos peixes. $\mathrm{O}$ momento de ingestão foi registrado usando-se uma câmera de vídeo digital instalada sobre o aquário. Após a alimentação, as sobras do alimento foram retiradas e pesadas para estimar a quantidade ingerida por diferença. Os conteúdos estomacais foram pesados após sa crificar os peixes em intervalos pós-prandiais (pós-alimentar) de duas horas. Os intervalos de tempo máximos usados foram planejados para evitar a digestão total do alimento, cujo tempo pós-prandial não poderia ser medido.
Assim foram utilizados tempos pós-prandiais máximos que permitissem a digestāo de aproximadamente 90\% do alimento inicial (Swenson \& Smith, 1973).

Após o sacrifício, o comprimento padrão (medidos até $0,1 \mathrm{~cm}$ ) e o peso total (pesados até $0,01 \mathrm{~g}$ ) do peixe foram registrados. $\mathrm{O}$ peso seco do conteúdo estomacal foi obtido após secagem em estufa com temperatura de $50^{\circ} \mathrm{C}$ por uma semana. Através das equaçōes de correção do peso úmido para o peso seco foram calculados os pesos secos iniciais dos itens alimentares ingeridos.

A redução do conteúdo estomacal com o tempo pós-prandial foi analisada em conjunto com outras variáveis usando uma análise de covariância. As variáveis independentes foram: tipo de alimento (variável categórica), peso da piranha, peso inicial do alimento, tempo pósprandial e temperatura. A variável resposta foi a quantidade de alimento no estômago em porcentagem. Como a relação entre processos metabólicos e temperatura são geralmente exponenciais, optou-se por transformar a variável resposta para logaritmo antes de realizar as análises.

As equações finais foram ajustadas por regressão múltipla. O teste de Bonferroni foi utilizado para testar se haviam diferenças significativas entre as taxas de evacuação gástrica dos três itens alimentares e entre as temperaturas. O teste de Student foi usado para comparar as constantes das regressões com o valor teórico de $100 \%$ para testar o efeito da secagem do conteúdo estomacal sobre o ajuste da regressão.

\section{RESULTADOS}

Foram feitos 96 testes independentes com P. nattereri no período de 4 meses. Os 
exemplares apresentaram comprimento padrão variando de 11,5 a $17,0 \mathrm{~cm}$ e peso total variando de 70 a $230 \mathrm{~g}$; todos foram caracterizados como indivíduos adultos.

Tempos pós-prandiais de até 14 horas foram suficientes para obter um esvaziamento estomacal de $87 \%$ para o músculo ingerido. Tempos pós-prandiais mais longos não foram testados, pois nos intervalos adotados (2h) a
A quantidade de alimento no estômago variou significativamente com as interaçōes entre o tempo pós-prandial e o tipo de alimento, e entre a temperatura e o tipo de alimento (Tab. 1). Isto indica que a taxa de evacuação gástrica (alimento no estômago / tempo pós-prandial) e a redução do alimento no estômago com a temperatura, dependeram do tipo de alimento oferecido. $\mathrm{O}$ peso da piranha $(\mathrm{p}=0,854)$, o peso inicial do

Tabela 1. Análise de co-variância da temperatura, tempo pós-prandial, tipo de alimento, peso inicial do alimento e peso do predador. Três tipos de alimento foram testados: músculo e nadadeira de peixe e gafanhoto.

\begin{tabular}{|c|c|c|c|c|c|}
\hline Fatores & Soma de quadrados & GL & Média de quadrados & $\bar{F}$ & $\overline{\mathbf{P}}$ \\
\hline Temperatura & 1,545 & 1 & 1,545 & 87,830 & $<0.001$ \\
\hline Tempo pós-prandial & 39,657 & 1 & 39,657 & $2.254,737$ & $<0.001$ \\
\hline Tipo de alimento & 0,411 & 2 & 0,206 & 11,694 & $<0,001$ \\
\hline Peso inicial do alimento & 0,001 & 1 & 0,001 & 0,043 & 0,837 \\
\hline Peso do predador & 0,001 & 1 & 0,001 & 0,034 & 0,854 \\
\hline Tipo de alimento * Temperatura & 0,484 & 2 & 0,242 & 13,757 & $<0,001$ \\
\hline Tipo de alimento * peso inicial do alimento & 0,063 & 2 & 0,032 & 1,797 & 0,171 \\
\hline |Tipo de alimento * peso do predador & 0,065 & 2 & 0,033 & 1,859 & 0,162 \\
\hline Tipo de alimento * tempo pós-prandial & 0,815 & 2 & 0,044 & 2,509 & $<0,001$ \\
\hline
\end{tabular}

quantidade de alimento restante seria $0 \%$ (vazio) e poderia viciar a estimativa dos coeficientes. Tempos pós-prandiais máximos de 10 horas estimaram em média $65 \%$ do esvaziamento estomacal dos itens nadadeira e gafanhoto. A inclusão de tempos prandiais mais longos (14h) alteraram a distribuição dos resíduos e as análises estatísticas foram excluídas das análises. alimento $(p=0,837)$, e suas interações com o tipo de alimento $(p=0,162$ e $p=0,171$, respectivamente), não tiveram efeito significativo na variável resposta (Tab. 1). Regressões múltiplas foram então calculadas para cada tipo de alimento, usando apenas as duas principais variáveis: tempo pós-prandial e temperatura (Tab. 2). A análise dos resíduos

Tabela 2. Dados das análises das regressões múltiplas entre músculo e nadadeira de $\mathrm{S}$. insignis e gafanhoto (Paulinia sp,) a sobra estomacal após o tempo $(\mathrm{Y})$. Tp = tempo pós - prandial e $\mathrm{T}=$ temperatura.

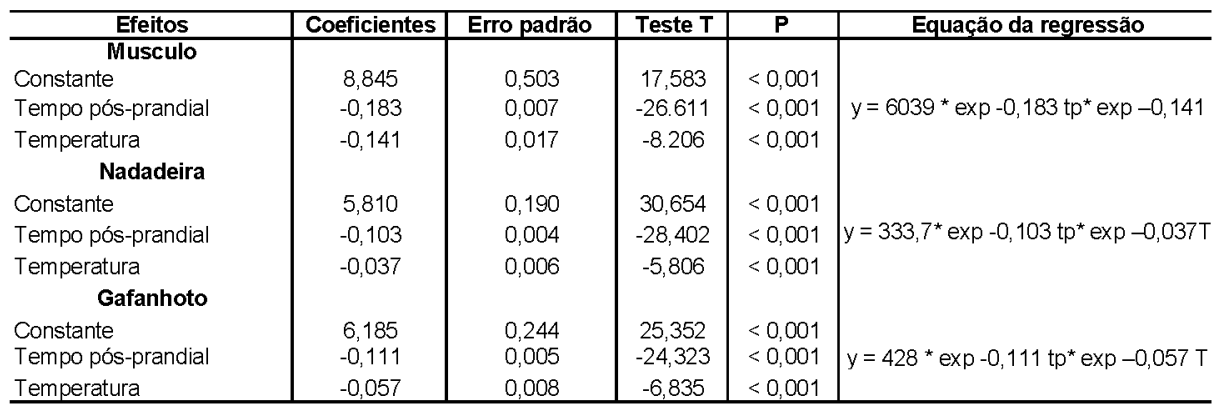




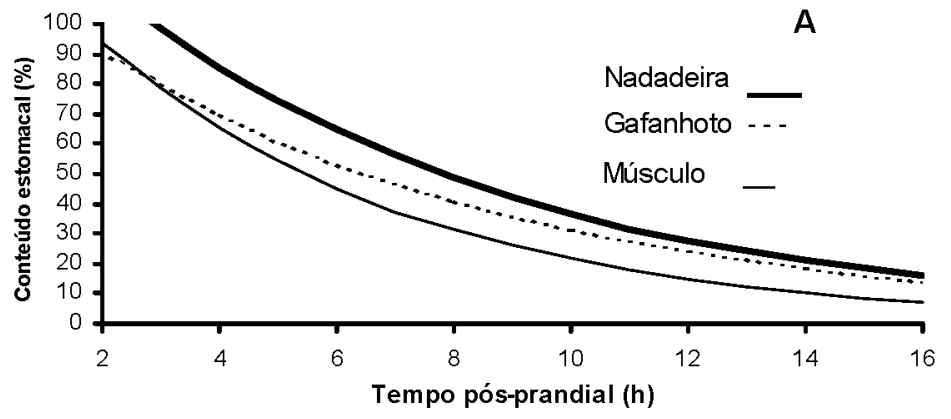

B
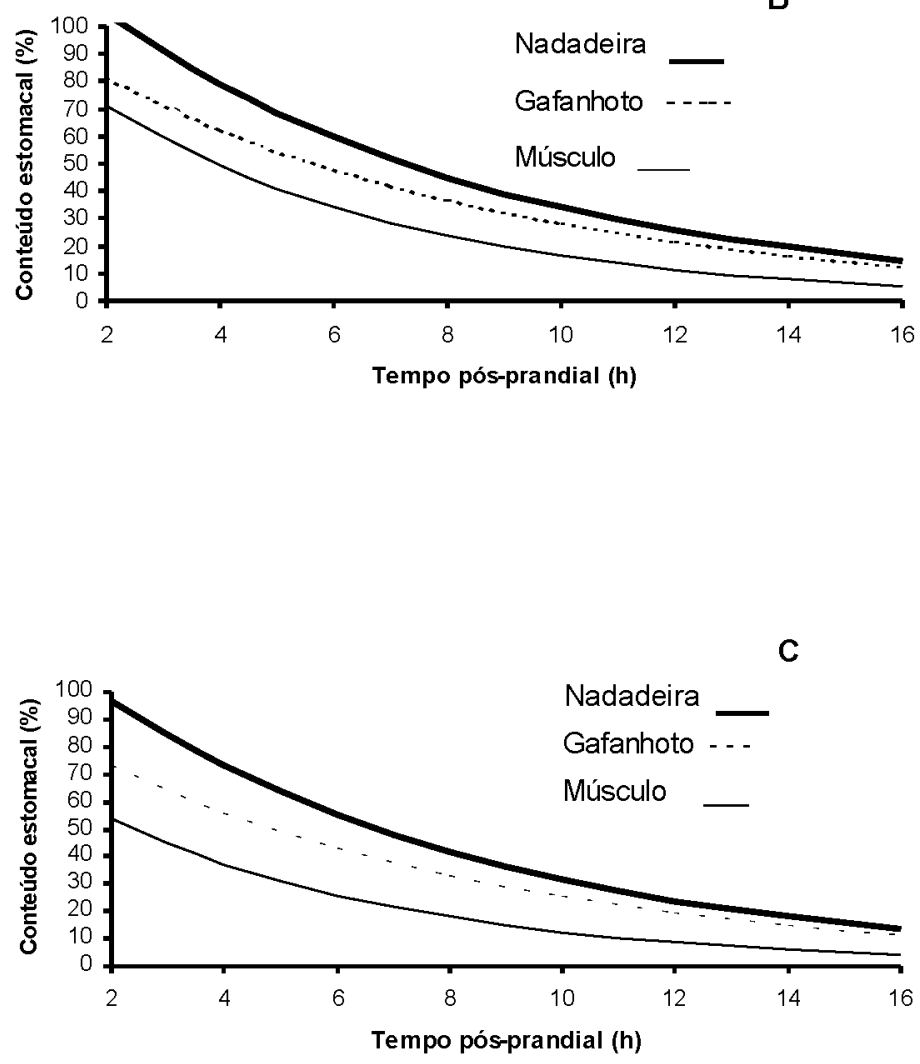

Figura 1. Velocidade de passagem do alimento pelo estômago de Pygocentrus nattereri, na temperatura de 27 C - A; 29C - B e 31C - C 
de músculo, nadadeira e gafanhoto mostraram bom ajuste no modelo exponencial (Figura 1).

O coeficiente do tempo pós-prandial na regressão para músculo foi significativamente inferior aos coeficientes das regressões de nadadeira e gafanhoto (teste de Bonferroni; $\mathrm{t}=$ 10,$0 ; \mathbf{t}=7,2 ;$ g.l. $=64 ; \mathrm{p}<0,05$, respectivamen te) (Tab. 2). Os coeficientes encontrados para nadadeira e gafanhoto não foram significativamente diferentes. Assim a taxa de evacuaçāo gástrica do músculo foi maior do que os outros dois itens. Músculos foram digeridos a uma taxa de $18,3 \% \mathrm{~h}^{-1}$, enquanto que nadadeiras e gafanhotos a uma taxa média de $10,7 \% \mathrm{~h}^{-1}$. $\mathrm{O}$ coeficiente da temperatura na regressão para músculo também foi significativamente diferente dos coeficientes obtidos com nadadeira e gafanhoto (teste de Bonferroni; $\mathrm{t}=5,7 ; \mathrm{t}=4,6$, g.1.=65; $\mathrm{p}<0,005)$. Porém, não ocorreu diferença significativa para nadadeira e gafanhoto $(t=1,8)$. Assim o efeito da temperatura sobre o tempo de passagem do músculo pelo estômago foi mais acentuado (taxa média de passagem do alimento de $14,1 \%{ }^{\circ} \mathrm{C}$ ) do que com nadadeira e gafanhoto de $4,5 \%{ }^{\circ} \mathrm{C}$.

As constantes das regressões para músculo, nadadeira e inseto apresentaram valores de 8,845, 5,810 e 6,185 (Tab. 2). Esses valores em peso úmido foram comparados através do teste de Student com 4,605, referente ao valor esperado de $100 \%$. As constantes da equação com músculo $(t=7,02)$, nadadeira $(t=2,51)$ e gafanhoto $(t=4,87)$, foram significativamente superiores a $100 \%$.

A mesma comparação foi feita usando constantes calculadas das regressões múltiplas, onde o peso úmido $(\mathrm{Pu})$ foi substituído pelo peso seco (Ps). Os valores de peso seco foram calculados com as regressões abaixo:

$$
\text { Ps }=0,62 \mathrm{Pu}-0,198 ; \mathrm{r}^{2}=0,9259 \text {; músculo }
$$$$
\text { Ps }=0,8243 \mathrm{Pu}-0,17 ; \mathbf{r}^{2}=0,953 \text {; }
$$
nadadeira

$$
\text { Ps }=0,898 \mathrm{Pu}-0,0498 ; \mathbf{r}^{2}=0,947 ;
$$
gafanhoto.

As regressões de peso seco de múscu lo, nadadeira e gafanhoto apresentaram cons tantes de $9,48,7,07$, e 5,60 , respectivamente. Semelhante as constantes calculadas com peso úmido, estas também foram significativamente diferentes do valor esperado de 100\%; tmusculo $=5,91$ e t nadadeira $=4,3$, exceto em gafanhoto $(\mathrm{t}=1,43)$.

\section{DISCUSSÃO}

A passagem do alimento pelo estômago depende da temperatura, tempo pós-prandial, tipo e tamanho do alimento, peso, tamanho e fisiologia do predador e grau de estresse (MacDonald et al., 1982; Jobling, 1987; Bromley, 1994; Salvanes et al., 1995). Apesar de ser influenciada por todos estes parâmetros, no presente experimento foram testados apenas cinco deles: temperatura, tempo pósprandial, tipo de alimento, peso do predador e peso do alimento. O peso do alimento e o peso do predador não foram relevantes, pois não tiveram efeito na massa de alimento presente no estômago. Já a temperatura, o tempo pós prandial e o tipo do alimento estiveram relacionadas com a digestāo em $P$. nattereri.

O tipo de alimento consumido pela piranha caju pode levar a diferentes velocidades de evacuação. O tempo de 14 horas pós-alimentar explicou o esvaziamento gástrico de cerca de $90 \%$ do alimento a $27^{\circ} \mathrm{C}$, quando este era músculo de peixe. Por outro lado, nadadeira e gafa nhoto, demonstraram tempo de esvaziamento de 19 horas (estimados da regressão) para al cançarem $10 \%$ do peso inicial. Portanto músculo de peixe foi evacuado em menor tempo do que nadadeira e gafanhoto (5 horas a mais que músculo). A taxa de evacuação gástrica do músculo foi $18,0 \% \mathrm{~h}-1$, enquanto que em nadadeira 
e gafanhoto foram de $10,5 \% \mathrm{~h}-1$. Essa diferença poderia ser explicada pelo tipo de estrutura do músculo, que possui menor rigidez que os demais itens, podendo apresentar menor resistência à digestão.

Resultados semelhantes foram encontrados com vários tipos de alimentos. Jones (1974) observou que itens que possuem exoesqueleto duro (carapaças de crustáceos ou insetos), demoravam mais para serem evacuados do que presas com menor rigidez. Segundo este autor, o efeito do exoesqueleto (constituído de quitina) aciona uma barreira para penetração do suco digestivo e retarda a digestāo do alimento, diminuindo a taxa de evacuação gástrica. Bromley (1991) também observou tempos diferencia dos de evacuação para dois tipos de alimento (peixe e lagosta), provavelmente devido aos tipos de estruturas diferentes que estes dois itens possuem. Poliquetos (menos rígido) foram digeridos mais rápido que anfípodos e bivalves (quitinosos e mais rígido) (MacDonald et al., 1982). Krill e pedaços de moluscos foram digeridos mais rápidos que poliquetos (Nelson \& Ross, 1995). A rápida digestāo em krill deve-se ao leve exoesqueleto que este possui. Segundo este autor, as diferenças encontradas na diges tâo nos diferentes tipos de presas (poliquetos, krill, moluscos e peixe) consumidos por Raja erinacea pode estar relacionado com a superfície do tecido (camada da epiderme) ou endoesqueleto, e que, consequientemente, estariam influenciando na velocidade de passagem do alimento.

Os resultados encontrados com piranha caju concordam bem com os expostos acima. Nadadeiras, que possuem partes ossificadas e gafanhotos que possuem exoesqueleto quitinoso deveriam ser evacuados mais lentamente do que músculo de peixe que oferece menor resistência a digestāo.
A influência da temperatura sobre a digestāo é bem demonstrada (E1liot, 1972; Persson, 1979; Bromley, 1994; Nelson \& Ross, 1995). A temperatura age controlando o metabolismo dos peixes, interferindo no processo digestivo e tendo também importante efeito na entrada e saída de alimento (Pandian \& Vivekanandan, 1985).

De uma forma geral, o aumento na temperatura leva a um aumento na digestāo. No presente estudo, a temperatura influenciou na taxa de evacuação gástrica acelerando o tempo de passagem dos itens alimentares testados. Em músculo, o efeito da temperatura sobre o tempo de passagem pelo estômago foi mais acentuado do que os efeitos da temperatura pelo tempo de passagem em nadadeira e gafanhoto. Outros estudos relacionaram o efeito da temperatura sobre os diferentes tipos de alimentos, concluindo que ambos são importantes parâmetros que afetam a digestão e a velocidade de evacuação (Jones, 1974; Tseitlin, 1980; Marques et al., 1993; Bromley, 1994; Temming \& Herrmann, 2001; Koed, 2001).

E comum as regressões de alimento pelo tempo pós-prandial estimarem valores superiores a $100 \%$ de alimento no tempo zero (Tyler, 1970). Os motivos para esta discrepância podem ser: 1) existência de fases estacionárias no início da digestão causada pelo período de inanição (Rosch, 1987) ou; 2) a grande quantidade de suco gástrico agindo sobre o alimento e aumentando o seu peso. A segunda alternativa foi testada em $P$. nattereri substituindo o alimento úmido pelo alimento seco no cálculo da regressão.

Neste caso a ausência de suco gástrico, também estimou valores superiores a 100\% no tempo zero, como em peso úmido. Apenas o peso seco em gafanhoto estimou corretamente o valor teórico de $100 \%$ do alimento. Assim é 
pouco provável que este efeito seja causado pelo excesso de suco gástrico. A hipótese de uma fase estacionária no início da digestão poderia explicar as constantes altas, mas esta não foi testada aqui e deve ser considerada em estudos futuros. A taxa de evacuação gástrica em P. nattereri, pode ser considerada elevada. Dependendo do tipo de alimento a piranha tem uma velocidade de digestão rápida, variando de acordo com o item alimentar e a temperatura. O tempo total estimado de evacuação através das regressões foi inferior a 14 horas para músculo e 19 horas para nadadeira e gafanhoto a $27^{\circ} \mathrm{C}$. Marques et al., (1993) com Pseudoplatystoma corruscans, estimaram a evacuaçāo gástrica até 10\% do conteúdo inicial em aproximadamente 15 horas na temperatura de $25^{\circ} \mathrm{C}$. Rabelo (1999), com Cichla monoculus, estimou a evacuaçāo gástrica até $10 \%$ em 16 horas na temperatura média da água de $27^{\circ} \mathrm{C}$. Peixes de regiôes temperadas como Salmo trutta, Perca fluviatilis e Sebastes melanops, evacuam seus estômagos em até 76 horas na temperatura média de $11^{\circ} \mathrm{C}$ (Fange \& Grove, 1979; Persson, 1979; Brodeur, 1984).

Na temperatura média diurna dos lagos de várzea $\left(29^{\circ} \mathrm{C}\right)$ a piranha caju digere músculo ainda mais rápido, esvaziando seu estômago em 12 horas para músculo e 17 horas para nadadeira e gafanhoto. Esta velocidade de digestāo pode explicar em parte a voracidade da piranha caju.

Dois modelos matemáticos são normalmente utilizados para descrever a evacuação gástrica: o modelo exponencial e o modelo linear. A utilização de equações exponenciais implica que porcentagens iguais de alimento passam em intervalos de tempo iguais. Entretanto quando quantidades constantes de alimento passam pelo estômago em intervalos regulares, o processo é melhor descrito por uma equação linear, o que significa que a cada intervalo a um aumento de porcentagem de alimento evacuado. A escolha de um ajuste ou outro pode depender de características alimentares ou de aspectos teóricos. Jobling (1987), argumenta que se o alimento consiste de itens pequenos e de fácil digestão, o modelo de evacuação adequado seria exponencial. Porém, se o alimento é constituído de grandes itens de alto conteúdo energético e difícil digestāo, o melhor modelo seria o linear. O argumento de Jobling (1987) aparentemente não se aplica a todos os casos. Nelson \& Ross (1995), por exemplo, analisaram o ajuste de quatro tipos de alimentos na mesma temperatura e encontraram que a digestão de pequenos itens foi melhor descrita pelo modelo linear. Isto sugere que a escolha do ajuste depende também de outras variáveis que não aquelas assumidas por Jobling (1987).

No presente estudo, o modelo utilizado foi o exponencial, pois uma única equaçāo incluiu o efeito do tempo de passagem e a temperatura, e geralmente processos enzimáticos envolvendo a temperatura são melhor ajustados por esse modelo. De forma semelhante Brett \& Higgs (1970) e Elliot (1972) observaram em seus estudos que a relação entre a temperatura e evacuação gástrica era exponencial.

\section{AGRADECIMENTOS}

Ao Instituto Nacional de Pesquisas da Amazônia. Ao CNPq através de uma bolsa de mestrado. A cooperação Brasil/Espanha através do PPI- 3020. Ao Autor Dr. Carlos Araújo Lima pela orientação.

\section{BIBLIOGRAFIA CITADA}

Beyer, J. M.; Lucchetti, G.; Gray, G. 1988. Digestive tract evacuation in northern squafish (Ptychocheilus oregonensis). Can. J. Fish. Aquat. Sci., 4 (3): 548-553. 
Bittencourt, M.M. 1994. Aspectos da demografia e do ciclo de vida de Pygocentrus nattereri Kner, 1860 em um lago de várzea da Amazônia Central (Lago do Rei, Ilha do Careiro). Tese de doutorado. INPA/UFAM, Manaus, p. 205.

Brett, J.R.; Higss, D.A . 1970. Effect of temperature on the rate of gastric digestion In fingerling sockeye salmon, Oncorhyncus nerka. J. Fish. Res. Bd Can 27: 1767 - 1779.

Brodeur, R.D. 1984. Gastric evacuation rates for two foods in the black rockfish Sebastes melanops Girard. Journal of Fish Biology., 24: 287-298.

Bromley, P. J. 1991. Gastric evacuation in cod (Gadus morhua L.) . In: Daan, N. \& Feffenwine, M. P., Multispecies Models relevant to Management of living resourses. (eds), pp. 93-98. Copenhagen: International council for the exploration of the Sea, Symposium 193.

Bromley, P. J. 1994. The role of gastric evacuation experiments in quantifying the feeding rates of predatory fish. Reviews in Fish Biology and Fisheries 4: 36-66.

Elliott, J. M. 1972. Rates of gastric evacuation in brown trout, Salmo trutta L. Freshwater Biology.2: 1-18.

Fange, R.; Grove, D. J. 1979. digestion. In: Hoar, W.S. Randall, D. J. \& Brett, J. R. (eds). Fish Physiology VIII. London: Acad. Press, 1979. p. $61-260$.

Figueiredo, G.; Vieira, J. 1998. Cronologia alimentar e dieta da corvina, Micropogonias furnieri, no estuário da lagoa do Patos, RS, Brasil. Atlântica.20:.55-72.
Fink, W.L. 1993. Revision of the piranha genus Pygocentrus (Teleostei, Characiformes). Copeia $665-687$.

Foxx, R.M. 1972. Attack preferences of the red bellied piranha (Serrasalmus nattereri). Animal Behaviour., 20: 280-283

Glenn, C. L.; Ward, F. J. 1968. "Wet" weight as a method for measuring stomach contents of walleyes, Stizostedion vitreum vitreum. J. Fish. Res. Bd. Canada, 25 (70): 1505 - 1507.

Jobling, M. 1981. Mathematical models of gastric emptying and the estimation of daily rates of food consumption for fish. Journal of Fish Biology. 19:245-257.

Jobling, M. 1987. Influences of food particle size and dietary energy content on patterns of gastric evacuation in fish test of a physiological model of gastric emptying. Journal of Fish Biology. 30: 299-314.

Jones, R. 1974. The rate of elimination of food from the stomachs of haddock Melanogrammus aeglefinus, cod Gadus morhua, and whiting Merlangius merlangus. Journal du Conseil, Conseil international pour l'exploration de la mer 35: 225-243.

Koed, A. 2001. The effects of meal size, body size and temperature on gastric evacuation in pikeperch. Journal of Fish Biology. 58: 281290.

MacDonald, J.S.; Waiwood, K.G. \& Green, R.H. 1982. Rates digestion of different prey in Atlantic Cod (Gadus morhua), ocean pout (Macrozoarces amercanus), winter flounder (Pseudopleuronectes americanus), and American Plaice (Hippoglossoides platessoides). Can. J. Fish. Aquat. Sci. 39 (5): 651-659. 
Machado - Allison, A.; Garcia, C. 1986. Food habits and morphological changes during ontogeny in three Serrasalmin fish species of Venezuelan floodplains. Copeia, 1: 193196.

Magnuson, J.J. 1969. Digestion and food consumption by skipjack tuna (Katsuwonus pelamis). Trans. Am. Fish. Soc., 98 (3): 379 392.

Marques, E.E.; Agostinho, A. A.; Sampaio, A. A.; Agostinho, S.C. 1993. Alimentação, Evacuação gástrica e Cronologia da digestão de jovens de Pintado Pseudoplatystoma corruscans (Siluriformes, Pimelodidae) e suas relações com a temperatura ambiente. Revista Unimar. 14: 207-221.

Merona, B.; Bittencourt. 1993. Les peuplements de poissons du "Lago do Rei, um lac d'inodation d'Amazonie centrale: description générale. Amazoniana. XII (3/4): 415-441.

Nelson, G. A.; Ross, M. R. 1995. Gastric evacuation in little skate. Journal of Fish Biology. 46:.977-986.

Pandian, T. J.; Vivekanandan, E. 1985. Energetics of feeding and digestion. In Tytler, P. \& Calow, P. (eds). Fish energetics: new perspectives. London: Croom Helm. P 99 124.

Pauly, D. 1994. Quantitative analysis of published data on the growth, metabolism, food comsumption, and related features of the red-bellied piranha, Serrasalmus nattereri (Characidae). Environmental Biology of Fishes. 41: 423-437.

Persson, L. 1979. The effects of temperature and different food organisms on the rate of gastric evacuation in perch (Perca fluviatilis). Freshwater Biology.9:.99-104.
Rabelo, H. 1999. A dieta e o consumo diário de alimento de Cichla monoculus na Amazônia Central. Dissertação de mestrado. INPA FUA, Manaus, Am. 41p.

Rosch, R. 1987. Effect of experimental conditions on the stomach evacuation of Coregonus lavaretus L. Journal of Fish Biology 30: 521 - 531.

Sainsbury, K. J. 1986. Estimation of food consumption from field observations of fish feeding cycles. Journal of fishes Biology. 29: 23-26.

Saint - Paul, U.; Zuanon, J. 2000. Fish communities in central Amazonian White and Blackwater floodplains. Environmental Biology of Fishes. 53:

$235-250$.

Salam, A.; Davies, P. M. C. 1994. Effect of body weight and temperature on the maximum daily food consumption of Esox lucius. Journal of Fish Biology. 44: 165-167.

Salvanes, A. G. V.; Aksnes, D. L.; Giske, J. 1995. A surface-dependent gastric evacuation model for fish. Journal of Fish Biology. 47:.679-695.

Smith, R. R. 1989. Nutritional energetics. In Halver, J. E. (eds). Fish Nutrition. Academic Press, San Diego. pp. 2-31.

Swenson, W. A.; Smith Jr, L. L. 1973. Gastric digestion, food consumption, feeding peridicity, and food conversion eficiency in walleye (Stizostedion vitreum vitreum). Journal of Fisheries Research Board of Canada. 30:.1327-1336.

Temming, A.; Herrmann, J. P. 2001. Gastric evacuation of horse mackerel. II. The effects 
of different prey types on the evacuation model. Journal of Fish Biology. 58: 12461256.

Tseitlin, V.B. 1980. Duration of gastric digestion in fishes. Mar. Ecol., Prog. Ser., 2 (4): 277-280.

Tyler, A. V. 1970. Rate of gastric emptying in young cod. J. Fish. Res. Bd Can. 27: 1177 $-1189$.

Submetido à publicação: 06/03/2000

Aceito: $29 / 10 / 2002$ 